Perinatology pISSN 2508-4887 • elSSN 2508-4895
Sung Hwan Choi, MD, Seung Han Shin, MD, Ee-Kyung Kim, MD, PhD, Han-Suk Kim, MD, PhD

Department of Pediatrics, Seoul National University College of Medicine, Seoul, Korea
Received: 15 August 2018

Revised: 14 September 2018

Accepted: 16 October 2018

Correspondence to

Seung Han Shin, MD

Department of Pediatrics, Seoul

National University College of

Medicine, 101 Daehak-ro, Jongno-gu,

Seoul 03080, Korea

Tel: $+82-2-2072-7230$

Fax: +82-2-747-5130

E-mail: revival421@snu.ac.kr

Copyright@ 2019 by The Korean Society of Perinatology

This is an Open Access article distributed under the terms of the Creative Commons Attribution Non-Commercial License (http://creativecommons.org/ license/by-nc/4.0/), which permits unrestricted non-commercial use, distribution, and reproduction in any medium, provided that the original work is properly cited.

\section{Respiratory Outcomes at 12 Months of Corrected Age of Preterm Infants with Severe Bronchopulmonary Dysplasia Re- quiring Protracted Invasive Ventilation}

Objective: The objective of this study was to describe respiratory and neurodevelopmental outcomes in infants with severe bronchopulmonary dysplasia (BPD) who needed invasive ventilation until 36 weeks' postmenstrual age (PMA).

Methods: A retrospective observational single-center study was conducted in our hospital. Eighty preterm infants born between January 2007 and December 2016 with less than 28 weeks' gestational age and classified as having severe BPD were included in the study. Patients with invasive ventilation at 36 weeks' PMA (invasive group) were compared with those with noninvasive ventilation (noninvasive group) in terms of perinatal characteristics and postnatal outcomes.

Results: Antenatal characteristics and basic patient characteristics were comparable between the two groups. Incidence of pulmonary hemorrhage (13.6 vs. 1.7\%, $P=0.061$ ) and clinical sepsis (66.7 vs. $31.0 \%, P=0.004)$ was more in the invasive group. Invasive group had longer hospital stay (133.50 \pm 104.52 vs. $114.00 \pm 24.71$ days, $P=0.031$ ), higher rates of readmission due to respiratory problems before 12 months of corrected age ( 57.1 vs. $32.1 \%, P=0.045$ ), higher rates of having a tracheostomy (22.7 vs. $1.7 \%, P=0.005$ ), and higher rates of infants with respiratory support at a corrected age of 6 months (22.7 vs. $3.5 \%, P=0.016)$. Neurodevelopmental outcomes including Bayley Scales of Infant Development-III, cerebral palsy, hearing aid, blindness, and composite outcome of them revealed no differences between the two groups.

Conclusion: Invasive ventilation until postmenstrual age of 36 weeks does not predict poorer neurodevelopmental outcomes in infants with severe BPD. However, the invasive group was more prone to develop respiratory problems after discharge.

Key Words: Preterm infants, Bronchopulmonary dysplasia, Respiration, Artificial, Growth and Development, Lung injury

\section{Introduction}

Bronchopulmonary dysplasia (BPD) is a well-known risk factor for poor clinical and neurological outcomes in preterm infants. Since the introduction of definition of BPD by the National Institute of Child Health and Human Development (NICHD) in the year 2000, ${ }^{1}$ there have been several reports on the correlations between BPD severity and clinical or neurodevelopmental outcomes in preterm infants. ${ }^{2,3}$

Among very preterm infants, the infants with severe BPD comprise a heterogeneous group in terms of respiratory support and relevant postnatal outcomes. ${ }^{4}$ This can be attributed to NICHD definition, which simply defines severe BPD as need for 30\% or more of supplemental oxygen and/or positive pressure at 36 weeks' postmenstrual age (PMA), irrespective of the ventilation method. Such a heterogeneity could consequently lead to 
various clinical and neurodevelopmental outcomes in infants with severe BPD.

Recent studies have suggested that the BPD definition needs modification considering the long-term outcomes. Brumbaugh et al. ${ }^{5}$ reported that there was no independent effect of severity of BPD on the cognitive function of preterm infants. Malavolti et al. ${ }^{6}$ suggested that BPD diagnosis at 40 weeks' PMA rather than 36 weeks' PMA might allow for better prediction of neurodevelopmental outcomes at 2 years of age. Furthermore, the need for protracted mechanical ventilation is known to be associated with poorer postnatal outcomes and neurodevelopmental impairments. ${ }^{7,8}$

The objective of this study was to determine whether invasive ventilation until 36 weeks' PMA among patients with severe BPD predicts poorer clinical and neurodevelopmental outcomes.

\section{Methods}

\section{Study subjects}

A retrospective observational study was conducted in $\mathrm{Neo}^{-}$ natal Intensive Care Unit (NICU) of Seoul National University Children's Hospital. Preterm infants who were born at less than 28 weeks' gestational age between January 2007 and December 2016 and were diagnosed as severe BPD were included in the study. Infants who were born in other hospitals, those who died or were transferred before 36 weeks' PMA, or those who had any congenital or chromosomal anomalies were excluded from the study.

Severe BPD was defined as a need for positive-pressure respiratory support and/or $\mathrm{FiO}_{2} 0.3$ or more at 36 weeks' PMA. ${ }^{1}$ In case of the use of humidified high-flow nasal cannula (HHFNC), any respiratory support with a flow rate higher than $4 \mathrm{~L} /$ min at 36 weeks' PMA was considered to be severe BPD. ${ }^{9-11}$ Study population was categorized into two groups: those required invasive ventilation (invasive group), and those who did not require invasive ventilation (noninvasive group) at 36 weeks' PMA.

\section{Data collection}

Z-scores of birth weight, length, and head circumference were calculated using the Fenton preterm growth chart. ${ }^{12}$ The Z-scores of anthropometric data after 50 weeks' PMA were calculated using the WHO Child Growth Standards. ${ }^{13}$ Small for gestational age was defined as birth weight and/or length percentile less than 10 percentile of Fenton preterm growth chart.

Patent ductus arteriosus (PDA) was defined as any ductus arteriosus with echocardiographic evidence that required treatment. Presence of bacteria in lower respiratory tract for each patient was recorded using tracheobronchial aspirate regardless of Gram stain characteristics. ${ }^{14,15}$ Clinical sepsis was defined as symptoms and signs of infection that prompted the need for antibiotic treatment for more than 3 days. ${ }^{16}$ Clinical signs and symptoms of infection included at least one from each of the three categories: general parameters (fever, hypothermia, apnea/tachypnea, other signs of respiratory distress, significant edema or positive fluid balance, hyperglycemia), inflammatory parameters (leukocytosis/leukopenia, increased serum C-reactive protein, increased serum procalcitonin level), and hemodynamic parameters (hypotension, tachycardia, decreased capillary refill time or mottling, acute oliguria, increased base deficit). Among the patients who fulfilled the criteria for clinical sepsis, proven sepsis was defined if bacterial growth in at least 1 blood culture is confirmed. Respiratory support at the time of discharge and at 6 months of corrected age (CA) were defined as need for any supplemental oxygen and/or positivepressure support.

Maternal data and antenatal characteristics of each patient such as maternal age, preeclampsia, preterm premature rupture of membrane (PPROM), the time interval between PPROM and delivery, prenatal antibiotics, histologic chorioamnionitis, oligohydramnios, gestational diabetes mellitus, antenatal steroid, mode of delivery, and multiplicity were also collected.

\section{Neurodevelopmental follow-up}

Patients were assessed at a CA of 8 months and 18 months, using the Bayley Scales of Infant Development, third edition (BSID-III). Serial follow-up of the neurodevelopmental status by rehabilitation specialists was started at 2 months of CA. Cerebral palsy was diagnosed by rehabilitation specialists according to international standards. ${ }^{17}$ Data about the need for a hearing aid and blindness of infants were also collected. For BSID-III, infants without available data were excluded in the 
analyses. A composite outcome of neurodevelopmental impairment was defined as any component of BSID-III less than 70, cerebral palsy, bilateral blindness, or bilateral hearing impairment at 18 month of CA.

\section{Statistical analyses}

T-tests for continuous data and chi-square tests for categorical data were used in the statistical analysis. $P$-value less than 0.05 was considered statistically significant in all the analyses. Multiple logistic regression analysis was used for correcting the effect of confounding factors on neurodevelopmental outcomes. All of the statistical analyses were conducted with SPSS version 21 (SPSS Inc, Chicago, IL, USA).

\section{Results}

There was a total of 327 preterm infants who were born at less than 28 weeks' gestation during the study period. After excluding one infant born with Down syndrome and 66 infants who died before 36 weeks' PMA, there were 246 preterm infants with BPD who were included in the study; 80 of these infants were classified as having severe BPD with 22 infants intubated and invasively ventilated and 58 infants not intubated

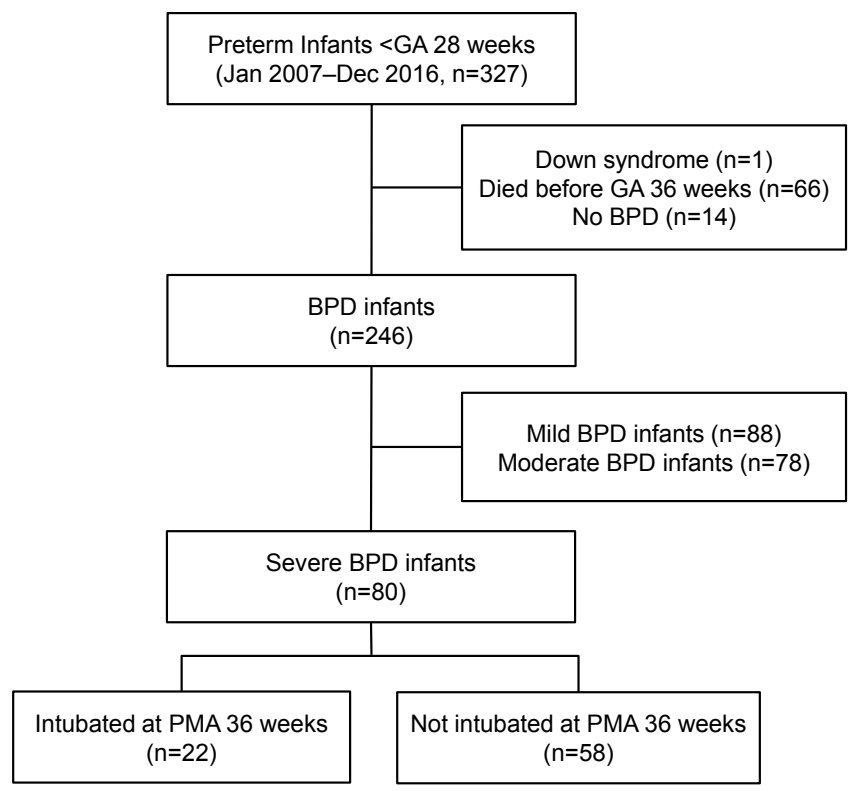

Fig. 1. Flow diagram of the preterm infants involved in the study. GA, gestational age; BPD, bronchopulmonary dysplasia; PMA, postmenstrual age. at 36 weeks' PMA (Fig. 1). Of those infants who were not intubated at 36 weeks' PMA, 35 infants (60.34\%) were supported by HHFNC, 17 infants (29.31\%) by nasal continuous positive airway pressure, three infants $(5.17 \%)$ by noninvasive neutrally adjusted ventilatory assist, and three infants (5.17\%) by nasal prong, respectively. There were no significant differences in antenatal characteristics between the invasive group and the noninvasive group (Table 1).

Gestational age, anthropometric data at birth, and the percentage of small-for-gestational age infant were similar between the two groups. Incidence of respiratory distress syndrome was comparable between the two groups, but incidence of pulmonary hemorrhage was slightly more in the invasive group with borderline significance ( 13.6 vs. $1.7 \%, P=0.061$ ). The invasive group was more likely to receive systemic dexamethasone for BPD treatment (61.9 vs. 21.4\%, $P=0.001$ ). However, the total dose of systemic dexamethasone per body weight $(1.26 \pm 1.72$ vs. $0.98 \pm 0.56 \mathrm{mg} / \mathrm{kg}, P=0.320)$ and PMA at dexamethasone exposure (223.5 \pm 24.13 vs. $222.83 \pm 43.12$ days, $P=0.966)$ were not significantly different between the two groups. There was no difference in the incidence of PDA and presence of bacteria in the tracheobronchial aspirate between the two groups. Clinical sepsis (66.7\% vs. 31.0\%, $P=0.004)$ and proven sepsis (52.4\% vs. $22.4 \%, P=0.011$ ) were more prevalent in the invasive group than in the noninvasive group. Neonatal outcomes such as intraventricular hemorrhage (IVH),

Table 1. Antenatal Characteristic of the Study Population

\begin{tabular}{lccc}
\hline Characteristic & Invasive $(\mathrm{n}=22)$ & Noninvasive $(\mathrm{n}=58)$ & $P$-value \\
\hline Maternal age (years) & $32.50 \pm 3.25$ & $33.00 \pm 5.80$ & 0.260 \\
Preeclampsia & $2(9.1)$ & $4(6.9)$ & 0.665 \\
PROM & $13(59.1)$ & $38(65.5)$ & 0.593 \\
PROM duration* (days) & $14.00 \pm 12.00$ & $11.00 \pm 18.17$ & 0.823 \\
Prenatal antibiotics & $18(85.7)$ & $44(75.9)$ & 0.537 \\
Chorioamnionitis & $12(54.5)$ & $34(58.6)$ & 0.742 \\
Oligohydramnios & $6(27.3)$ & $15(26.3)$ & 0.931 \\
GDM & $0(0.0)$ & $3(5.2)$ & 0.557 \\
Antenatal steroid & $14(63.6)$ & $40(69.0)$ & 0.650 \\
Cesarean section & $10(45.5)$ & $36(62.1)$ & 0.180 \\
Multiple births & $13(59.1)$ & $32(55.2)$ & 0.752 \\
\hline
\end{tabular}

Values are presented as mean \pm standard deviation or number (\%).

Abbreviations: PROM, preterm premature rupture of membrane; GDM, gestational diabetes mellitus.

*The time interval between PROM and delivery. 
periventricular leukomalacia (PVL), and necrotizing enterocolitis (NEC) were comparable in the two groups (Table 2).

Body weight Z-score in the invasive group at 36 weeks' PMA

Table 2. Characteristics of Study Population

\begin{tabular}{lccc}
\hline Characteristic & $\begin{array}{c}\text { Invasive } \\
(\mathrm{n}=22)\end{array}$ & $\begin{array}{c}\text { Noninvasive } \\
(\mathrm{n}=58)\end{array}$ & $\begin{array}{c}P \text { - } \\
\text { value }\end{array}$ \\
\hline Gestational age (days) & $178.50 \pm 8.93$ & $179.00 \pm 9.83$ & 0.752 \\
\hline Body weight (g) & $740 \pm 166.25$ & $765 \pm 168.71$ & 0.551 \\
\hline Male sex & $15(68.2)$ & $31(53.4)$ & 0.234 \\
\hline Body weight at birth (Z-score) & $0.01 \pm 0.93$ & $-0.10 \pm 0.86$ & 0.982 \\
\hline Length at birth (Z-score) & $0.00 \pm 1.07$ & $-0.06 \pm 0.99$ & 0.243 \\
\hline Head circumference at birth (Z-score) & $-0.18 \pm 0.79$ & $-0.23 \pm 1.41$ & 0.707 \\
\hline Small for gestational age & $5(22.7)$ & $7(12.1)$ & 0.295 \\
\hline Apgar score at 1 minute & $2.50 \pm 1.85$ & $3.00 \pm 1.71$ & 0.721 \\
Apgar score at 5 minutes & $4.50 \pm 1.79$ & $5.00 \pm 1.93$ & 0.237 \\
Respiratory distress syndrome & $20(90.9)$ & $49(84.5)$ & 0.718 \\
Pulmonary hemorrhage & $3(13.6)$ & $1(1.7)$ & 0.061 \\
\hline Air leak & $3(13.6)$ & $3(5.4)$ & 0.342 \\
\hline Systemic dexamethasone use & $13(61.9)$ & $12(21.4)$ & 0.001 \\
\hline Total dexamethasone dose* (mg/kg) & $1.26 \pm 0.72$ & $0.98 \pm 0.56$ & 0.320 \\
\hline PDA requiring treatment & $20(90.9)$ & $48(82.8)$ & 0.495 \\
Presence of bacteria from LRT & $20(90.9)$ & $55(94.8)$ & 0.612 \\
Clinical sepsis & $14(66.7)$ & $18(31.0)$ & 0.004 \\
Proven sepsis & $11(52.4)$ & $13(22.4)$ & 0.011 \\
\hline IVH grade $\geq 3$ & $2(9.1)$ & $11(19.0)$ & 0.498 \\
Periventricular leukomalacia & $3(14.3)$ & $7(12.1)$ & 0.721 \\
\hline NEC grade $\geq 2 A$ & $6(27.3)$ & $8(13.8)$ & 0.192 \\
\hline Va & & & \\
\hline
\end{tabular}

Values are presented as mean \pm standard deviation or number (\%).

Abbreviations: PDA, patent ductus arteriosus; LRT, lower respiratory tract; IVH, intraventricular hemorrhage; NEC, necrotizing enterocolitis.

*Total dose of systemic dexamethasone for the treatment of BPD divided by the body weight at the time of dexamethasone administration. was higher than that of the noninvasive group $(-1.23 \pm 0.89$ vs. $-1.83 \pm 0.67, P=0.030$ ). However, the invasive group required

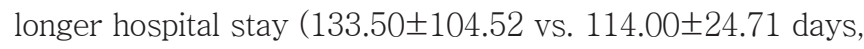
$P=0.031$ ). The duration of invasive ventilation was significantly longer in the invasive group ( $83.94 \pm 16.07$ vs. $52.04 \pm 16.26$ days, $P=0.000$ ) (Table 3, Fig. 2).

Patients of the invasive group had higher rates of readmission due to respiratory problems before 12 months of CA (57.1 vs. $32.1 \%, P=0.045)$. Need for a tracheostomy was more required in the invasive group (22.7 vs. $1.7 \%, P=0.005$ ), but there was no significant difference in the need of respiratory support at the

Table 3. Postneonatal Outcomes and Growth of Study Population

\begin{tabular}{lccc}
\hline Postneonatal outcome & $\begin{array}{c}\text { Invasive } \\
(\mathrm{n}=22)\end{array}$ & $\begin{array}{c}\text { Noninvasive } \\
(\mathrm{n}=58)\end{array}$ & $\begin{array}{c}P \text { - } \\
\text { value }\end{array}$ \\
\hline Duration of admission (days) & $133.50 \pm 104.52$ & $114.00 \pm 24.71$ & 0.031 \\
Duration of invasive ventilation (days) & $83.94 \pm 16.07$ & $52.04 \pm 16.26$ & 0.000 \\
Respiratory support at discharge & $15(71.4)$ & $35(60.3)$ & 0.367 \\
Tracheostomy & $5(22.7)$ & $1(1.7)$ & 0.005 \\
Readmission due to respiratory & $12(57.1)$ & $18(32.1)$ & 0.045 \\
problems before CA 12 months & & & \\
Respiratory support at CA 6 months & $5(22.7)$ & $2(3.5)$ & 0.016 \\
Death after PMA 36 weeks & $1(2.5)$ & $1(1.7)$ & 0.477 \\
Body weight at PMA 36 weeks (Z-score) & $-1.23 \pm 0.89$ & $-1.83 \pm 0.67$ & 0.030 \\
Length at PMA 36 weeks (Z-score) & $-2.12 \pm 1.08$ & $-2.49 \pm 0.70$ & 0.363 \\
Head circumference at PMA 36 weeks & $-2.01 \pm 0.94$ & $-2.44 \pm 0.91$ & 0.095 \\
(Z-score) & & & \\
\hline Body weight at CA 12 months (Z-score) & $-0.58 \pm 1.57$ & $-0.63 \pm 1.45$ & 0.325 \\
Length at CA 12 months (Z-score) & $-0.07 \pm 1.94$ & $-0.52 \pm 1.30$ & 0.566 \\
Head circumference at CA 12 months & $-0.88 \pm 2.83$ & $-1.27 \pm 1.41$ & 0.785 \\
(Z-score) & & & \\
\hline
\end{tabular}

Values are presented as mean \pm standard deviation or number (\%).

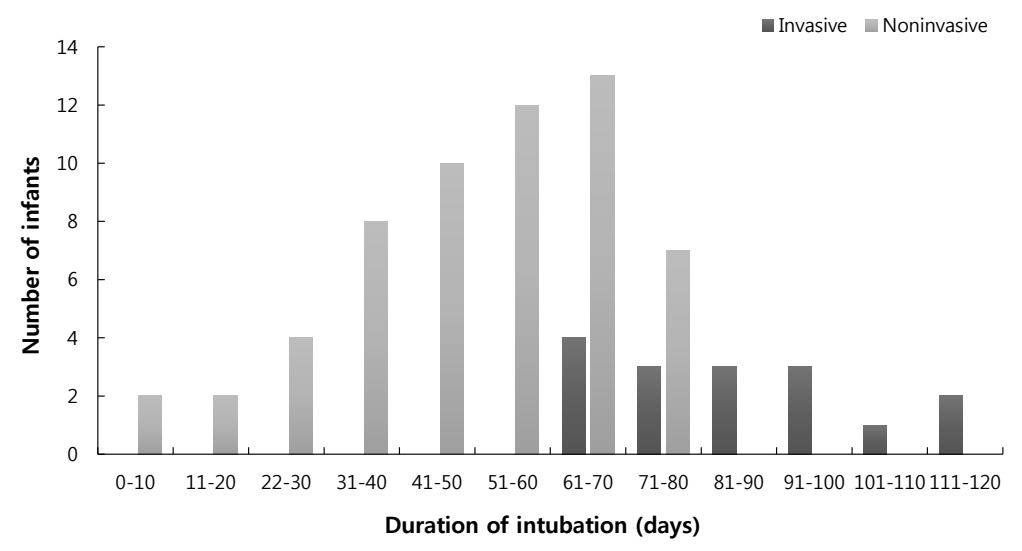

Fig. 2. Duration of invasive ventilation. 
time of discharge. All 35 infants in the noninvasive group who needed respiratory support at discharge were supported with supplemental oxygen by nasal prong. In the invasive group, 11 infants (73.3\%) were supported by nasal prong, and two infants (13.3\%) were supported by home ventilator via tracheostomy. One infant in the invasive group was discharged with a tracheostomy and oxygen without positive-pressure ventilation.

At 6 months of $\mathrm{CA}$, respiratory support rate was higher in the invasive group (22.7\% vs. $3.5 \%, P=0.016$ ) (Table 3 ). Two infants of the noninvasive group were supported by nasal prong; of the five infants of the invasive group, two infants were supported by nasal prong, one infant by home ventilator via a tracheostomy, one infant by a tracheostomy and oxygen without positive-pressure ventilation, and one infant by highfrequency oscillatory ventilation via an endotracheal tube, respectively.

BSID-III data was available for 51 infants (63.8\%) at 8 months of CA, and 40 infants (50.0\%) at 18 months of CA. There were no significant differences in the neurodevelopmental outcomes

\section{Table 4. Neurodevelopmental Outcomes of Study Population}

\begin{tabular}{lccc}
\hline $\begin{array}{l}\text { Neurodevelopmental } \\
\text { outcome }\end{array}$ & Invasive $(\mathrm{n}=22)$ & Noninvasive $(\mathrm{n}=58)$ & $P$-value \\
\hline Cerebral palsy & $1(5.0)$ & $5(8.9)$ & 1.000 \\
Hearing aid & $1(5.0)$ & $2(3.5)$ & 1.000 \\
\hline Blindness & $0(0.0)$ & $0(0.0)$ & - \\
\hline BSID-III composite score & & & \\
\hline Cognition (CA 8 months) & $82.50 \pm 15.35$ & $91.74 \pm 11.44$ & 0.052 \\
\hline Language (CA 8 months) & $86.50 \pm 17.36$ & $89.21 \pm 6.71$ & 0.721 \\
\hline Motor (CA 8 months) & $81.17 \pm 21.59$ & $89.84 \pm 13.85$ & 0.206 \\
\hline Cognition (CA 18 months) & $87.22 \pm 11.48$ & $88.55 \pm 14.56$ & 0.803 \\
\hline Language (CA 18 months) & $78.44 \pm 8.86$ & $87.17 \pm 15.25$ & 0.112 \\
\hline Motor (CA 18 months) & $84.44 \pm 18.71$ & $84.97 \pm 15.06$ & 0.932 \\
\hline BSID-III score <70 & & & \\
Cognition (CA 8 months) & $1(12.5)$ & $1(2.3)$ & 0.292 \\
\hline Language (CA 8 months) & $1(16.7)$ & $0(0.0)$ & 0.176 \\
\hline Motor (CA 8 months) & $2(33.3)$ & $1(3.1)$ & 0.059 \\
\hline Cognition (CA 18 months) & $1(20.0)$ & $4(12.9)$ & 1.000 \\
\hline Language (CA 18 months) & $1(11.1)$ & $4(13.3)$ & 1.000 \\
\hline Motor (CA 18 months) & $2(22.2)$ & $4(13.3)$ & 0.607 \\
NDI at CA 18 months* & $2(18.2)$ & $10(28.6)$ & 0.701 \\
\hline Values & &
\end{tabular}

Values are presented as mean \pm standard deviation or number (\%).

Abbreviations: CA, corrected age; BSID-III, Bayley Scales of Infant Development, third edition.

${ }^{*}$ Cerebral palsy or hearing aid or blindness or any BSID-III score less than 70.
(Table 4). Multiple logistic regression analysis adjusting for gestational age (odds ratio [OR], 0.988; 95\% confidence interval [CI], 0.984-0.997), IVH grade 3 or 4 (OR, 3.558; 95\% CI, 0.578-21.901), systemic dexamethasone use (OR, 0.730; 95\% CI, 0.083-6.416), proven sepsis (OR, 2.062; 95\% CI, 0.309-13.739), PVL (OR, 3.238; 95\% CI, 0.510-20.558), and NEC (OR, 2.676; 95\% CI, 0.375-19.099) showed that invasive ventilation at 36 weeks' PMA was not associated with neurodevelopmental impairment at 18 months of CA (OR, 0.424; 95\% CI, 0.034-5.221).

\section{Discussion}

Recently, there have been efforts to revise the definition of $\mathrm{BPD}$ in preterm infants because $\mathrm{BPD}$ is a complex disease with multifactorial pathogenesis. ${ }^{18-20}$ A more practical and working definition predicting the long-term outcome is required to identify the candidate population for treatment or prophylaxis of BPD. In the present study, preterm infants with severe BPD requiring invasive ventilation at PMA 36 weeks had more respiratory morbidities than those who did not require invasive ventilation.

The two groups showed no significant difference in the rate of respiratory support at the time of discharge from NICU. However, the readmission rate due to respiratory problems and the need for respiratory support at CA of 6 months were higher in the invasive group. This reflects the difference in the severity of BPD between these two groups, which could also be deduced by the difference in the rate of systemic dexamethasone use for BPD treatment. Similar results were reported in another study, in which it was seen that among preterm infants with severe BPD, those who required both oxygen and assisted ventilation at 36 weeks' PMA showed the highest readmission rate among study population. ${ }^{21}$

The need for invasive respiratory support until 36 weeks' PMA did not predict poorer neurodevelopmental outcomes in infants with severe BPD in the present study. This is in disagreement to various studies that have reported the association of the severity of BPD and neurodevelopmental outcomes. Walsh et al. ${ }^{7}$ demonstrated that duration of mechanical ventilation was a risk factor for neurologic impairment, the odds of impairment 
increasing by a factor of 1.18 per week of ventilation. Vliegenthart et al. ${ }^{22}$ demonstrated that the strategy of restricted invasive mechanical ventilation when started immediately after the delivery was associated with improved neurodevelopmental outcomes. However, the median duration of invasive ventilation in these studies was not longer than that seen in the present study. Lodha et $a .^{23}$ demonstrated that BPD infants with chronic oxygen dependency did not show higher neurodevelopmental disability rates than those without chronic oxygen dependency.

Walsh et al. ${ }^{7}$ reported that neurologic impairment started to increase when infants remain ventilated for more than 28 days. However, the risk of death or neurological impairment was not associated with duration of invasive ventilation for those infants who were ventilator dependent for more than 60 days. Data from Canadian Neonatal Network also showed similar results in terms of serious neurosensory impairment in infants with oxygen only and infants with oxygen and respiratory support at 36 weeks' PMA. ${ }^{18}$ Interestingly, putting the timing of severity assessment off till 44 weeks of PMA showed no discrimination in prediction of serious neurosensory impairment in that study. Therefore, refining the definition of severe BPD for the better prediction of neurodevelopment might not be much useful.

Postnatal dexamethasone for BPD treatment has been reported to have detrimental effects on neurodevelopment of preterm infants. ${ }^{24,25}$ In this study, the rate of systemic dexamethasone use was higher in the invasive group. However, the total dose of systemic dexamethasone per body weight was equivocal between the two groups, which might balance out the confounding effect of dexamethasone use on neurodevelopmental outcomes. Wilson-Costello et al. ${ }^{26}$ showed that neurodevelopmental impairment increased with higher dose of postnatal corticosteroid.

The invasive group had higher body weight Z-score at 36 weeks' PMA. However, both group showed comparable growth at 12 months of CA. Such a relative decline of growth in the invasive group might be attributable to severity of BPD in this group of infants. BPD has been reported to be a strong predictor of growth failure. ${ }^{27,28}$ Moreover, the present study did not collect or analyze the data of nutritional intervention after discharge in the study population.

We acknowledge some limitations as well. Firstly, this was a single-center study with small sample size. Secondly, the association between the ventilation status at 36 weeks' PMA and neurodevelopmental outcomes were analyzed at a relatively short-term period.

In conclusion, infants with severe BPD who required invasive ventilation until 36 weeks' PMA showed comparable neurodevelopmental outcomes to infants with severe BPD who were supported by noninvasive ventilation at 36 weeks' PMA. However, the invasive group showed prolonged hospitalization, more frequent readmission due to respiratory problems, higher rate of tracheostomy, and higher rate of ventilator support at CA of 6 months. Further studies with large sample size evaluating the neurodevelopmental status of a later period might be required to characterize and categorize severe $\mathrm{BPD}$ more practically.

\section{Conflict of Interest}

No potential conflict of interest relevant to this article was reported.

\section{Acknowledgments}

This research was supported by Basic Science Research Program through the National Research Foundation of Korea (NRF) funded by the Ministry of Education (NRF-2017R1D1A1 B03036383).

\section{References}

1) Jobe AH, Bancalari E. Bronchopulmonary dysplasia. Am J Respir Crit Care Med 2001;163:1723-9.

2) Ehrenkranz RA, Walsh MC, Vohr BR, Jobe $A H$, Wright LL, Fanaroff $A A$, et al. Validation of the National Institutes of Health consensus definition of bronchopulmonary dysplasia. Pediatrics 2005;110:1353-60.

3) Short EJ, Kirchner HL, Asaad GR, Fulton SE, Lewis BA, Klein N, et al. Developmental sequelae in preterm infants having a diagnosis of bronchopulmonary dysplasia: analysis using a severity-based classification system. Arch Pediat Adol Med 2007;161:1082-7.

4) Guaman MC, Gien J, Baker CD, Zhang H, Austin ED, Collaco JM. Point prevalence, clinical characteristics, and treatment variation for infants 
with severe bronchopulmonary dysplasia. Am J Perinatol 2015;32:960-7.

5) Brumbaugh JE, Colaizy TT, Patel NM, Klein JM. The changing relationship between bronchopulmonary dysplasia and cognition in very preterm infants. Acta Paediatr 2018;107:1339-44.

6) Malavolti AM, Bassler D, Arlettaz-Mieth R, Faldella G, Latal B, Natalucci G. Bronchopulmonary dysplasia-impact of severity and timing of diagnosis on neurodevelopment of preterm infants: a retrospective cohort study. BMJ Paediatr Open 2018;2:e000165.

7) Walsh MC, Morris BH, Wrage LA, Vohr BR, Poole WK, Tyson JE, et al. Extremely low birthweight neonate with protracted ventilation: mortality and 18-month neurodevelopmental outcomes. J Pediatr 2005;146:708804.

8) Keszler M, Sant'Anna G. Mechanical ventilation and bronchopulmonary dysplasia. Clin Perinatol 2015;42:781-96.

9) Roberts CT, Owen LS, Manley BJ, Frøisland DH, Donath SM, Dalziel KM, et al. Nasal high-flow therapy for primary respiratory support in preterm infants. N Engl J Med 2016;375:1142-51.

10) Manley BJ, Owen LS, Doyle LW, Andersen CC, Cartwright DW, Pritchard $M A$, et al. High-flow nasal cannulae in very preterm infants after extubation. N Engl J Med 2013;369:1425-33.

11) Manley BJ, Dold SK, Davis PG, Roehr CC. High-flow nasal cannulae for respiratory support of preterm infants: a review of the evidence. Neonatology 2012;102:300-8.

12) Fenton $T R$, Kim JH. A systematic review and meta-analysis to revise the Fenton growth chart for preterm infants. BMC Pediatr 2013;13:59.

13) WHO Multicenter Growth Reference Study Group. WHO Child Growth Standards based on length/height, weight and age. Acta Paediatr 2006; 450:76-85

14) Beeton ML, Maxwell NC, Davies PL, Nuttall D, McGreal E, Chakraborty M, et al. Role of pulmonary infection in the development of chronic lung disease of prematurity. Eur Respir J 2011;37-1424-30.

15) Imamura T, Sato M, Go H, Ogasawara K, Kanai Y, Maeda H, et al. The microbiome of the lower respiratory tract in premature infants with and without severe bronchopulmonary dysplasia. Am J Perinatol 2017;34: 80-7.

16) Levy MM, Fink MP, Marshall JC, Abraham E, Angus D, Cook D, et al. 2001 SCCM/ESICM/ACCP/ATS/SIS International Sepsis Definitions Conference. Intensive Care Med 2003;29:530-8.

17) Palisano R, Rosenbaum P, Walter $S$, Russell D, Wood E, Galuppi B. Development and reliability of a system to classify gross motor function in children with cerebral palsy. Dev Med Child Neurol 1997;39:214-23.

18) Isayama T, Lee SK, Yang J, Lee D, Daspal S, Dunn M, et al. Revisiting the definition of bronchopulmonary dysplasia: effect of changing panoply of respiratory support for preterm neonates. JAMA Pediatr 2017;171: 271-9.

19) Jobe AH, Steinhorn R. Can we define bronchopulmonary dysplasia? J Pediatr 2017;188:19-23.

20) Bancalari E, Jain D. Bronchopulmonary dysplasia: can we agree on a definition? Am J Perinatol 2018;35:537-40.

21) Akangire G, Manimtim W, Nyp MF, Noel-MacDonnell J, Kays AN, Truog $W E$, et al. Clinical outcomes among diagnostic subgroups of infants with severe bronchopulmonary dysplasia through 2 years of age. Am J Perinatol 2018;35:1376-87.

22) Vliegenthart RJS, Onland W, Wassenaer-Leemhuis AG, De Jaegere APM, Aarnoudse-Moens CSH, van Kaam AH. Restricted ventilation associated with reduced neurodevelopmental impairment in preterm infants. Neonatology 2017;112:172-9.

23) Lodha A, Sauvé R, Bhandari $V$, Tang $S$, Christianson $H$, Bhandari A, et al. Need for supplemental oxygen at discharge in infants with bronchopulmonary dysplasia is not associated with worse neurodevelopmenta outcomes at 3 years corrected age. PLoS One 2014;9:e90843.

24) Qin G, Lo JW, Marlow N, Calvert SA, Greenough A, Peacock JL. Postnata dexamethasone, respiratory and neurodevelopmental outcomes at two years in babies born extremely preterm. PLoS One 2017;12:e0181176.

25) Cheong JL, Burnett AC, Lee KJ, Roberts G, Thompson DK, Wood SJ, et al. Association between postnatal dexamethasone for treatment of bronchopulmonary dysplasia and brain volumes at adolescence in infants born very preterm. J Pediatr 2014;164:737-43.e1.

26) Wilson-Costello D, Walsh MC, Langer JC, Guillet R, Laptook AR, Stoll BJ, et al. Impact of postnatal corticosteroid use on neurodevelopment at 18 to 22 months' adjusted age: effects of dose, timing, and risk of bronchopulmonary dysplasia in extremely low birth weight infants. Pediatrics 2009;123:e430-7.

27) Natarajan G, Johnson YR, Brozanski B, Farrow KN, Zaniletti I, Padula MA, et al. Postnatal weight gain in preterm infants with severe bronchopulmonary dysplasia. Am J Perinatol 2014;31:223-30.

28) Korhonen P, Hyödynmaa E, Lenko HL, Tammela O. Growth and adrena androgen status at 7 years in very low birth weight survivors with and without bronchopulmonary dysplasia. Arch Dis Child 2004;89:320-4. 